\title{
Psychosocial predictors of quality of life among chronic hemodialysis patients
}

\author{
Fahad D. Alosaimi, MD, Salem M. Alshahrani, Medical Student, Mohammed H. Aladhayani, Medical Student,
} Zaid K. Alwethenani, Medical Student, Mohammed J. Alsahil, Medical Student, Hasan A. Fadhul, MD.

\begin{abstract}
الأهداف : لفحص مدى ارتباط الاضطرابات النفسية الاجتماعية بجودة الحياة (QoL)

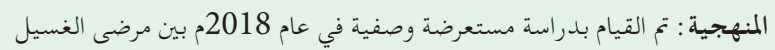

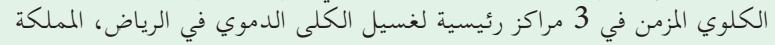

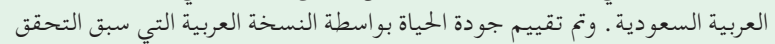

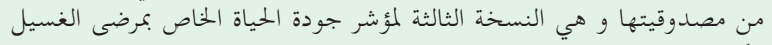

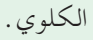

النتائج : تم ادراج ما مجموعه 101 مريض (56.0 (56 من الذكور و45 من الإناث ).

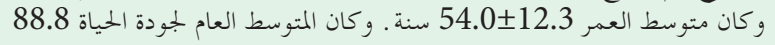

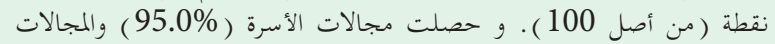

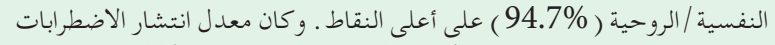

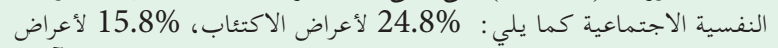

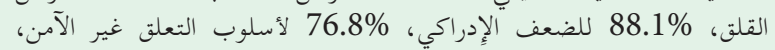

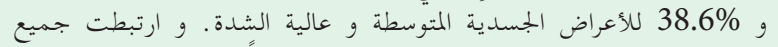

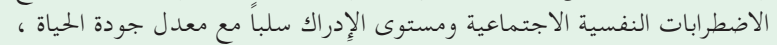

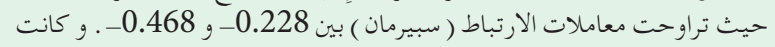

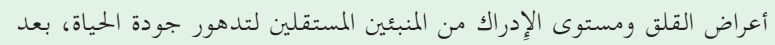

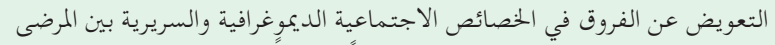

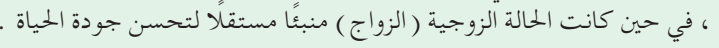

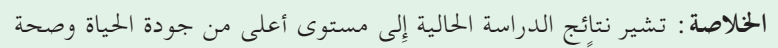

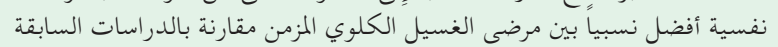

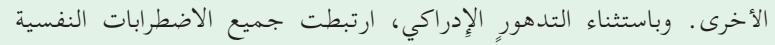

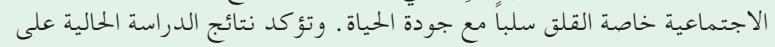

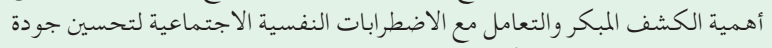
الحياة بين مرضى الغسيل الكملوي المزمن.
\end{abstract}

Objectives: To examine the association of several psychosocial disorders with quality of life (QoL) among chronic hemodialysis patients.

Methods: A cross-sectional observational study was conducted in 2018 and recruited chronic hemodialysis patients from 3 major hemodialysis centers in Riyadh, Kingdom of Saudi Arabia (KSA). Quality of life was assessed using a previously validated Arabic version of the dialysis version-III of the QoL index.
Results: A total of 101 patients $(56$ males and 45 females) were included. The mean age was $54.0 \pm 12.3$ years. The overall mean of QoL was $88.8 / 100$ points (out of 100$)$. The family $(95.0 \%)$ and psychological/spiritual (94.7\%) domains had the highest scores. The prevalence of psychosocial disorders was $24.8 \%$ for depressive symptoms, $15.8 \%$ for anxiety symptoms, $88.1 \%$ for cognitive impairment, $76.8 \%$ for insecure attachment style, and $38.6 \%$ for medium/high severity of somatic symptoms. All psychosocial disorders and higher cognition level were negatively correlated with QoL (Spearman correlation $r$ ranged between -0.228--0.468). After adjusting for sociodemographic and clinical characteristics, anxiety symptoms and higher cognition level were independent predictors of poor QoL, while marital status (married) was an independent predictor of good QoL.

Conclusion: We report higher QoL and relatively better psychosocial profiles among current chronic hemodialysis patients than reported before. Psychosocial disorders specially anxiety can negatively impact QoL. Patients who were not cognitively impaired were more negatively affected in QOL. The findings re-emphasize the importance of the early detection and management of psychosocial disorders to improve QoL in chronic hemodialysis patients.

Keywords: hemodialysis, quality of life, psychosocial, depression, anxiety, cognitive impairment, Saudi Arabia

Saudi Med J 2020; Vol. 41 (9): 990-998

doi: 10.15537/smj.2020.9.25271

From the Department of Psychiatry (Alosaimi, Fadhul), and from the College of Medicine (Alshahrani, Aladhayani, Alwethenani, Alsahil), King Saud University, Riyadh, Kingdom of Saudi Arabia.

Received 22nd March 2020. Accepted 23rd July 2020.

Address correspondence and reprint request to: Dr. Fahad D. Alosaimi, Department of Psychiatry, King Saud University, Riyadh, Kingdom of Saudi Arabia. E-mail: dr.fahad.alosaimi@gmail.com

ORCID ID: https://orcid.org/0000-0003-1027-5836 
$\mathrm{H}$ emodialysis is a lifesaving chronic therapy for an increasing number of patients with end-stage renal disease (ESRD). ${ }^{1}$ Researchers estimate that approximately 2 million patients worldwide receive chronic hemodialysis. ${ }^{2}$ The use of hemodialysis has been increasing and is expected to increase even more in the next decades, mainly due to aging of the population and increasing burden of contributing diseases such as diabetes and hypertension. ${ }^{2,3}$ This is expected to considerably increase the financial burden on the healthcare system, especially in developing countries. ${ }^{4}$ In Kingdom of Saudi Arabia (KSA), the prevalence and incidence of ESRD have been steadily increasing in recent decades. ${ }^{5}$ Similarly, the number of patients receiving hemodialysis increased from approximately 4,000 in 1995 to nearly 18,000 in 2017 .

In addition to the pathophysiological effects of ESRD and hemodialysis, patients also face significant lifestyle challenges that can increase the risk of developing depression, anxiety, cognitive impairment, and other psychosocial problems. ${ }^{7-9}$ The presence of these psychosocial disorders can negatively impact the course of the disease, including mortality and hospitalization. ${ }^{10,11}$ Additionally, several studies have suggested the negative impact of psychosocial disorders on different domains of quality of life (QoL) among chronic dialysis patients. ${ }^{12-14}$

As expected, chronic hemodialysis patients in KSA have poor QoL and a higher prevalence of depression and anxiety. ${ }^{15-18}$ Nevertheless, the high level of religiosity and spiritual coping among these patients may positively impact QoL. ${ }^{19}$ Additionally, it has been suggested that depression among these patients could be lower than similar patients in other parts of the world..$^{20}$ Although psychological symptoms have been examined in relation to hemodialysis adaptation, no studies examined the impact of psychosocial disorders on QoL among chronic hemodialysis patients in KSA. ${ }^{21}$ This is especially interesting in KSA, which has a unique conservative culture with a profound role of religion and family support. The current study aimed to examine the association of psychosocial disorders, including depression, anxiety, cognitive impairment, insecure attachment style, and severe somatic symptoms, with QoL among chronic hemodialysis patients.

Methods. Official statistics points to the presence of 270 hemodialysis centers in KSA, with approximately

Disclosure. Authors have no conflict of interests, and the work was not supported or funded by any drug company.
$50 \%$ of them directly funded by the Ministry of Health. ${ }^{6}$ The current study was conducted among patients receiving maintenance hemodialysis at 3 major centers in Riyadh, KSA (King Saud University Medical City, Prince Sultan Military Medical City, and King Salman Center for Kidney Diseases). Approximately 700 patients are regularly receiving free hemodialysis at the 3 centers.

Study design. A cross-sectional observational study was conducted between January and July 2018. All required ethical approvals were obtained from the institutional review board at the Faculty of Medicine at King Saud University in Riyadh, KSA. Additionally, administrative approvals were obtained from the administrations of the 3 centers.

Population. Patientswere recruited using convenience sampling technique. Therefore, consecutive chronic hemodialysis patients at the 3 included centers were invited to join the study. Only those who were receiving hemodialysis for at least 3 years were included. Patients who were able to give informed consent irrespective of their gender were asked to fill a structured study questionnaire and to complete multiple psychosocial tools. Patients under 18 years of age and those who were not able to answer the questionnaire or psychosocial tools (such as those with blindness, deafness, or severe dementia) were excluded. Out of 165 invited, 101 completed the study (response rate 61.2\%).

Data collection. A structured study questionnaire was used in data collection covering sociodemographic characteristics, hemodialysis-related characteristics, comorbidity, and psychiatric history (supplementary material-1). Additionally, a number of validated psychological tools has been used to assess exposures and outcomes of the study (supplementary material-2).

Exposure and outcome definitions. Exposure measures included psychological tools: the 9-item patient health questionnaire (PHQ-9), hospital anxiety and depression (HAD) scale, experience in close relationships scale (ECR-M16), Montreal cognitive assessment (MoCA), and the patient health questionnaire-physical symptoms (PHQ-15). ${ }^{22-29}$ The outcome was assessed by QoL index covering 4 domains: health and functioning, socioeconomic, psychological/ spiritual, and family domains. ${ }^{30,31}$ The details of each tool including the cut points for the diagnosis are shown in supplementary material.

Statistical analysis. The data was presented as frequencies and percentages for categorical variables and means and standard deviations (SDs) for continuous variables. Significant differences in QoL index between groups defined by demographics, clinical characteristics, 
comorbidity, and psychosocial tools were tested using the Mann-Whitney test (for 2-level variables) and KruskalWallis test (for variables with more than 2 levels). Correlations between QoL index and the continuous version of the psychological measures were tested using Spearman correlation. Multivariate stepwise linear regression model was run to detect potential predictors for higher level of QoL index. The model included all variables associated with the overall QoL index $(p<0.05$ or $p<0.010)$ in univariate analysis. All $p$-values were 2 -tailed. A $p$-value $<0.05$ was considered significant. The Statistical Package for the Social Science (SPSS), version 23.0 (IBM Corp., Armonk, NY, USA) was used for all statistical analyses.

Results. The current analysis included a total of 101 patients (56 males and 45 females). The overall mean QoL index was 88.8/100 points (out of 100). More details about the scores of different domains of QoL are shown in Figure 1. The mean age was $54.0 \pm 12.3$ years, and $55.4 \%$ were males. Two-thirds $(67.3 \%)$ of patients were currently married, and they had a mean of $5.2 \pm 3.1$ children. The majority $(96.0 \%)$ of the patients were Saudi, $59.4 \%$ had less than secondary education, $26.0 \%$ were working, and only $15.2 \%$ had a family income greater than SR10,000. Of the demographic characteristics, being older $\geq 60$ years $(p=0.020)$, being married ( $p=0.036)$, and being housewives $(p=0.009)$ were significantly associated with higher level of overall QoL index. More details about demographic characteristics are shown in Table 1.

Approximately $62.4 \%$ of the patients were aware of the medical causes of their renal failure. Some

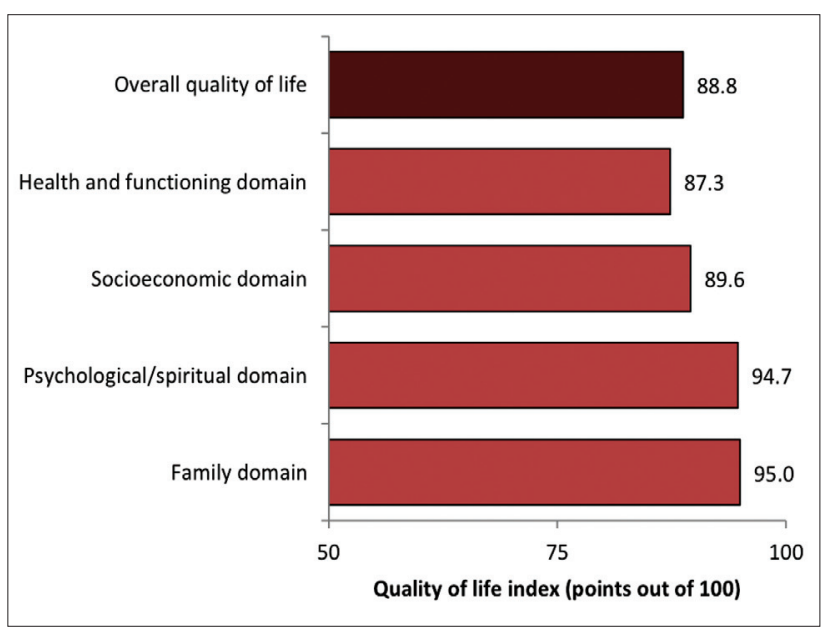

Figure 1 - Overall and domains of quality of life among chronic hemodialysis patients using the quality of life index (dialysis version-III).
Table 1 - Demographic characteristics of the chronic hemodialysis patients by the quality of life index.

\begin{tabular}{|c|c|c|c|}
\hline Characteristics & $\mathrm{n}(\%)$ & $\begin{array}{c}\text { Quality of life index } \\
\text { Mean } \pm \text { SD }\end{array}$ & $P$-value \\
\hline Age (years) Mean $\pm S D$ & $54.0 \pm 12.3$ & & \\
\hline $\begin{array}{l}18-44 \\
45-59 \\
\geq 60\end{array}$ & $\begin{array}{l}21(21.2) \\
42(42.4) \\
36(36.4)\end{array}$ & $\begin{array}{c}83.6 \pm 11.1 \\
89.3 \pm 10.9 \\
91.2 \pm 8.6\end{array}$ & 0.020 \\
\hline \multicolumn{4}{|l|}{ Gender } \\
\hline $\begin{array}{l}\text { Male } \\
\text { Female }\end{array}$ & $\begin{array}{l}56(55.4) \\
45(44.6)\end{array}$ & $\begin{array}{l}87.8 \pm 11.1 \\
89.9 \pm 9.6\end{array}$ & 0.276 \\
\hline \multicolumn{4}{|l|}{ Marital status } \\
\hline $\begin{array}{l}\text { Married } \\
\text { Single } \\
\text { Divorced } \\
\text { Widowed }\end{array}$ & $\begin{array}{c}68(67.3) \\
14(13.9) \\
9(8.9) \\
10(9.9)\end{array}$ & $\begin{array}{l}90.9 \pm 8.7 \\
84.0 \pm 11.4 \\
81.6 \pm 15.9 \\
87.4 \pm 11.2\end{array}$ & 0.036 \\
\hline \multicolumn{4}{|l|}{ Nationality } \\
\hline $\begin{array}{l}\text { Saudi } \\
\text { Non-Saudi }\end{array}$ & $\begin{array}{l}97(96.0) \\
\quad 4(4.0)\end{array}$ & $\begin{array}{l}88.8 \pm 10.4 \\
87.7 \pm 13.8\end{array}$ & $>0.99$ \\
\hline \multicolumn{4}{|l|}{ Educational status } \\
\hline $\begin{array}{l}\text { Less than secondary } \\
\text { Secondary } \\
\text { College or higher }\end{array}$ & $\begin{array}{l}60(59.4) \\
18(17.8) \\
23(22.8)\end{array}$ & $\begin{array}{c}89.9 \pm 9.6 \\
86.4 \pm 13.2 \\
87.6 \pm 10.3\end{array}$ & 0.327 \\
\hline \multicolumn{4}{|l|}{ Occupation } \\
\hline $\begin{array}{l}\text { Employed } \\
\text { Unemployed } \\
\text { Housewife }\end{array}$ & $\begin{array}{l}26(26.0) \\
48(48.0) \\
26(26.0)\end{array}$ & $\begin{array}{c}88.0 \pm 9.2 \\
87.2 \pm 11.6 \\
92.8 \pm 8.6\end{array}$ & 0.009 \\
\hline \multicolumn{4}{|l|}{ Monthly income } \\
\hline $\begin{array}{l}<5000 \\
5000-10000 \\
>10000\end{array}$ & $\begin{array}{l}56(56.6) \\
28(28.3) \\
15(15.2)\end{array}$ & $\begin{array}{c}88.2 \pm 10.8 \\
87.7 \pm 12.1 \\
91.6 \pm 4.2\end{array}$ & $>0.99$ \\
\hline \multicolumn{4}{|l|}{ Housing type } \\
\hline $\begin{array}{l}\text { Own } \\
\text { Rent } \\
\text { Provided by employer }\end{array}$ & $\begin{array}{c}69(68.3) \\
31(30.7) \\
1(1.0)\end{array}$ & $\begin{array}{l}90.1 \pm 9.5 \\
85.6 \pm 12.0 \\
95.9 \pm \mathrm{NA}\end{array}$ & 0.204 \\
\hline \multicolumn{4}{|c|}{ Number of household adults } \\
\hline $\begin{array}{l}\leq 5 \\
>5\end{array}$ & $\begin{array}{l}61(63.5) \\
35(36.5)\end{array}$ & $\begin{array}{c}87.6 \pm 12.0 \\
91.8 \pm 6.1\end{array}$ & 0.362 \\
\hline \multicolumn{4}{|l|}{ Regular exercise } \\
\hline $\begin{array}{l}\text { No } \\
\text { Yes }\end{array}$ & $\begin{array}{l}85(85.0) \\
15(15.0)\end{array}$ & $\begin{array}{c}89.3 \pm 9.9 \\
85.7 \pm 13.3\end{array}$ & 0.249 \\
\hline \multicolumn{4}{|l|}{ Current smoking } \\
\hline $\begin{array}{l}\text { No } \\
\text { Yes }\end{array}$ & $\begin{array}{l}90(89.1) \\
11(10.9)\end{array}$ & $\begin{array}{l}88.9 \pm 10.5 \\
87.8 \pm 10.9\end{array}$ & 0.526 \\
\hline \multicolumn{4}{|c|}{ Using alcohol or other substances } \\
\hline $\begin{array}{l}\text { No } \\
\text { Yes } \\
\end{array}$ & $\begin{array}{c}97(97.0) \\
3(3.0) \\
\end{array}$ & $\begin{array}{c}88.8 \pm 10.6 \\
86.8 \pm 6.9\end{array}$ & 0.278 \\
\hline
\end{tabular}

patients believed that psychological stress $(20.8 \%)$ and nonmedical causes such as evil eye/possession/black magic $(30.7 \%)$ could be a reason for their renal failure. The mean duration of hemodialysis was $9.9 \pm 6.8$ years. Approximately one-fourth $(26.7 \%)$ of the patients were using alternative medicine, such as herbs (63\%), religious ruqyah $(55.6 \%)$, and cauterization $(7.4 \%)$. The vast majority of the patients were satisfied with 
hemodialysis services provided by their center. The majority $(73.7 \%)$ of the patients reported receiving support from their families or friends, and only $23.8 \%$ had problems getting to the hemodialysis unit. Of the dialysis-related characteristics, only longer duration of hemodialysis was significantly associated with lower level of overall QoL index ( $p=0.027)$. More details about dialysis-related characteristics are shown in Table 2.

The majority $(90.1 \%)$ of the patients had comorbidity; with $38.5 \%$ of them suffer from 3 or more diseases. Only $3.0 \%$ of the patients reported having

Table 2 - Hemodialysis-related characteristics of the study participants by the quality of life index.

\begin{tabular}{|c|c|c|c|}
\hline Characteristics & \multicolumn{3}{|c|}{ Mean \pm SD } \\
\hline \multicolumn{4}{|c|}{ Knowledge of renal failure etiology } \\
\hline $\begin{array}{l}\text { No } \\
\text { Yes }\end{array}$ & $\begin{array}{l}38(37.6) \\
63(62.4)\end{array}$ & $\begin{array}{l}90.4 \pm 8.1 \\
87.8 \pm 11.6\end{array}$ & 0.557 \\
\hline \multicolumn{4}{|c|}{ Renal failure etiology $y^{*}$} \\
\hline $\begin{array}{l}\text { Hypertension } \\
\text { Diabetes } \\
\text { Hereditary } \\
\text { Renal atrophy } \\
\text { Medications } \\
\text { Inflammation } \\
\text { Other }\end{array}$ & $\begin{array}{rr}21 & (33.9) \\
5 & (8.1) \\
4 & (6.5) \\
5 & (8.1) \\
2 & (3.2) \\
4 & (6.5) \\
21 & (33.9)\end{array}$ & $\begin{array}{l}89.4 \pm 10.4 \\
86.5 \pm 12.2 \\
90.2 \pm 8.1 \\
87.1 \pm 10.8 \\
72.9 \pm 30.3 \\
82.9 \pm 11.6 \\
88.4 \pm 12.2\end{array}$ & $\begin{array}{c}0.387 \\
0.563 \\
0.772 \\
>0.99 \\
0.402 \\
0.180 \\
0.704\end{array}$ \\
\hline \multicolumn{4}{|c|}{ Family history of renal failure } \\
\hline $\begin{array}{l}\text { No } \\
\text { Yes }\end{array}$ & $\begin{array}{l}80(79.2) \\
21(20.8)\end{array}$ & $\begin{array}{l}89.4 \pm 10.0 \\
86.4 \pm 12.2\end{array}$ & 0.252 \\
\hline \multicolumn{4}{|c|}{ Role of psychological stress } \\
\hline $\begin{array}{l}\text { No } \\
\text { Yes }\end{array}$ & $\begin{array}{l}80(79.2) \\
21(20.8)\end{array}$ & $\begin{array}{l}89.3 \pm 10.5 \\
86.8 \pm 10.2\end{array}$ & 0.199 \\
\hline \multicolumn{4}{|c|}{ Role of evil eyelpossession/black magic } \\
\hline $\begin{array}{l}\text { No } \\
\text { Yes }\end{array}$ & $\begin{array}{l}70(69.3) \\
31(30.7)\end{array}$ & $\begin{array}{l}88.9 \pm 11.3 \\
88.5 \pm 8.5\end{array}$ & 0.330 \\
\hline \multicolumn{4}{|c|}{ Alternative medicine use } \\
\hline $\begin{array}{l}\text { No } \\
\text { Yes }\end{array}$ & $\begin{array}{l}74(73.3) \\
27(26.7)\end{array}$ & $\begin{array}{l}89.3 \pm 10.9 \\
87.2 \pm 9.2\end{array}$ & 0.078 \\
\hline \multicolumn{4}{|c|}{ Years of hemodialysis } \\
\hline $\begin{array}{l}\leq 5 \\
>5\end{array}$ & $\begin{array}{l}32(32.7) \\
66(67.3)\end{array}$ & $\begin{array}{l}92.1 \pm 8.4 \\
87.9 \pm 10.2\end{array}$ & 0.027 \\
\hline \multicolumn{4}{|c|}{ Satisfaction with provided hemodialysis } \\
\hline $\begin{array}{l}\text { Excellent } \\
\text { Good } \\
\text { Not bad } \\
\text { Bad }\end{array}$ & $\begin{array}{rr}87 & (86.1) \\
10 & (9.9) \\
1 & (1.0) \\
3 & (3.0)\end{array}$ & $\begin{array}{c}89.3 \pm 10.4 \\
86.3 \pm 9.6 \\
67.2 \pm \mathrm{NA} \\
86.9 \pm 10.2\end{array}$ & 0.251 \\
\hline \multicolumn{4}{|c|}{ Support from family or friends } \\
\hline $\begin{array}{l}\text { No } \\
\text { Yes }\end{array}$ & $\begin{array}{l}25(26.3) \\
70(73.7)\end{array}$ & $\begin{array}{c}85.4 \pm 15.1 \\
89.7 \pm 8.3\end{array}$ & 0.956 \\
\hline \multicolumn{4}{|c|}{ Problems getting to hemodialysis unit } \\
\hline $\begin{array}{l}\text { No } \\
\text { Yes }\end{array}$ & $\begin{array}{l}77(76.2) \\
24(23.8)\end{array}$ & $\begin{array}{l}89.5 \pm 10.3 \\
86.3 \pm 10.8 \\
\end{array}$ & 0.078 \\
\hline
\end{tabular}

psychiatric disorders before the diagnosis of renal failure. Only 6.9\% were regularly seeing a psychiatrist. Approximately $10.9 \%$ of the patients received a diagnosis of psychiatric disorders after the diagnosis of renal failure. Higher numbers of comorbidities $(p=0.004)$, specifically cardiovascular disease $(p=0.041)$ and hepatitis B $(p=0.030)$ and hepatitis C $(p=0.014)$, were significantly associated with lower level of overall QoL index. More details about medical and psychological comorbidities are shown in Table 3.

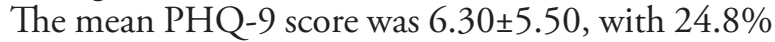
of the patients having depressive symptoms. The mean HAD anxiety score was $3.28 \pm 3.97$ and depression score was $4.06 \pm 3.91$, with $15.8 \%$ of the patients having anxiety and $19.8 \%$ of them having depressive symptoms. The mean ECR-M16 score was 3.38 \pm 1.42

Table 3 - Medical and psychological comorbidity of the chronic hemodialysis patients by the quality of life index.

\begin{tabular}{|c|c|c|c|}
\hline Variables & n (\%) & $\begin{array}{l}\text { Quality of life index } \\
\text { Mean } \pm \text { SD }\end{array}$ & $P$-value \\
\hline \multicolumn{4}{|c|}{ Number of comorbid diseases } \\
\hline $\begin{array}{l}<3 \\
\geq 3\end{array}$ & $\begin{array}{l}56(61.5) \\
35(38.5)\end{array}$ & $\begin{array}{l}91.3 \pm 8.2 \\
85.1 \pm 12.1\end{array}$ & 0.004 \\
\hline \multicolumn{4}{|l|}{ Comorbid diseases* } \\
\hline $\begin{array}{l}\text { Hypertension } \\
\text { Diabetes mellitus } \\
\text { Cardiovascular disease } \\
\text { Hepatitis C } \\
\text { Hyperlipidemia } \\
\text { Hyperthyroidism } \\
\text { Hepatitis B } \\
\text { Hypothyroidism } \\
\text { Asthma } \\
\text { Epilepsy } \\
\text { Others }\end{array}$ & $\begin{array}{rr}83 & (82.2) \\
37 & (36.6) \\
21 & (20.8) \\
12 & (11.9) \\
7 & (6.9) \\
6 & (5.9) \\
6 & (5.9) \\
5 & (5.0) \\
4 & (4.0) \\
3 & (3.0) \\
28 & (27.7)\end{array}$ & $\begin{array}{l}88.7 \pm 10.5 \\
89.3 \pm 10.9 \\
85.7 \pm 10.8 \\
81.6 \pm 11.6 \\
92.6 \pm 2.1 \\
86.8 \pm 7.6 \\
77.1 \pm 17.4 \\
86.4 \pm 10.5 \\
83.4 \pm 12.0 \\
90.4 \pm 9.7 \\
88.8 \pm 9.0\end{array}$ & $\begin{array}{l}0.883 \\
0.596 \\
0.041 \\
0.014 \\
0.904 \\
0.293 \\
0.030 \\
0.515 \\
0.184 \\
0.712 \\
0.491\end{array}$ \\
\hline \multicolumn{4}{|c|}{ Psychiatric disorder before the diagnosis of renal failure } \\
\hline $\begin{array}{l}\text { No } \\
\text { Yes }\end{array}$ & $\begin{array}{r}98(97.0) \\
3(3.0)\end{array}$ & $\begin{array}{l}88.8 \pm 10.5 \\
86.5 \pm 10.4\end{array}$ & 0.460 \\
\hline \multicolumn{4}{|c|}{ Change in psychological status after the diagnosis of renal failure } \\
\hline $\begin{array}{l}\text { No } \\
\text { Yes }\end{array}$ & $\begin{array}{l}58(57.4) \\
43(42.6)\end{array}$ & $\begin{array}{l}89.6 \pm 10.7 \\
87.6 \pm 10.1\end{array}$ & 0.110 \\
\hline \multicolumn{4}{|l|}{ Seeing a psychiatrist } \\
\hline $\begin{array}{l}\text { No } \\
\text { Yes }\end{array}$ & $\begin{array}{r}94(93.1) \\
7 \quad(6.9)\end{array}$ & $\begin{array}{l}88.8 \pm 10.7 \\
88.6 \pm 7.1\end{array}$ & 0.429 \\
\hline \multicolumn{4}{|c|}{ Diagnosed with psychiatric disorder } \\
\hline $\begin{array}{l}\text { No } \\
\text { Yes }\end{array}$ & $\begin{array}{l}90(89.1) \\
11(10.9)\end{array}$ & $\begin{array}{l}89.1 \pm 10.4 \\
86.0 \pm 11.0\end{array}$ & 0.376 \\
\hline \multicolumn{4}{|c|}{ Taking psychiatric medications } \\
\hline $\begin{array}{l}\text { No } \\
\text { Yes }\end{array}$ & $\begin{array}{l}1(12.5) \\
7(87.5)\end{array}$ & $\begin{array}{l}78.9 \pm \mathrm{NA} \\
90.0 \pm 8.0\end{array}$ & 0.500 \\
\hline
\end{tabular}


for anxiety and $2.73 \pm 1.21$ for avoidance, with $76.8 \%$ of the patients considered insecure. The mean MoCA cognitive function was $16.84 \pm 7.63$, with $88.1 \%$ of the patients having cognitive impairment (that is MoCA is less than 26 out of 30). The mean PHQ-15 score was $7.97 \pm 5.33$, with $38.6 \%$ of the patients having medium/high severity of somatic symptoms. All the above scores were significantly and negatively correlated with the level of overall QoL index ( $\mathrm{r}$ ranged between -0.228- -0.468). Patients who were not cognitively impaired were more negatively affected in QOL. More details about psychosocial examinations by the QoL index are shown in Table 4.

After adjusting for all the variables associated with overall QoL index, only the HAD anxiety score, MoCA score, and marital status were independent predictors of overall QoL index (Table 5). For each increase of one standard deviation in the HAD anxiety score and MoCA score (that is better cognition), there was an overall QoL index decrease of HAD anxiety score 0.425 and MoCA score 0.301 standard deviations. On the other hand, overall QoL index was 0.309 standard deviations higher in married patients than in non-married patients.

Discussion. The current study showed high levels of overall QoL among patients receiving hemodialysis at 3 centers in Riyadh, KSA. The QoL index tool used in the current study measured patient-perceived subjective QoL, while most of the previous studies among chronic dialysis patients focused on healthrelated QoL. ${ }^{13}$ While it is challenging to compare QoL measured using different tools in populations with different demographic and clinical characteristics, the current level of QoL appeared to be higher than those previously reported for chronic hemodialysis patients in KSA. ${ }^{15,16}$ Additionally, the current level of

Table 4 - Psychiatric examinations among the chronic hemodialysis patients by the quality of life index.

\begin{tabular}{|c|c|c|c|c|}
\hline Variables & n $\quad(\%)$ & $\begin{array}{l}\text { Quality of life index } \\
\qquad \text { Mean } \pm \text { SD }\end{array}$ & Spearman correlation & $P$-value \\
\hline PHQ-9 depression score & & $6.30 \pm 5.50$ & -0.251 & 0.011 \\
\hline $\begin{array}{l}\text { Normal }(0-9) \\
\text { Depression }(\geq 10)\end{array}$ & $\begin{array}{l}76(75.2) \\
25(24.8)\end{array}$ & $\begin{array}{l}90.1 \pm 8.8 \\
84.7 \pm 13.8\end{array}$ & & 0.207 \\
\hline$H A D$ anxiety score & & $3.28 \pm 3.97$ & -0.468 & $<0.001$ \\
\hline $\begin{array}{l}\text { Normal }(0-7) \\
\text { Anxiety }(>7)\end{array}$ & $\begin{array}{l}85(84.2) \\
16(15.8)\end{array}$ & $\begin{array}{l}90.2 \pm 9.4 \\
80.8 \pm 12.5\end{array}$ & & 0.007 \\
\hline HAD depression score & & $4.06 \pm 3.91$ & -0.270 & 0.006 \\
\hline $\begin{array}{l}\text { Normal }(0-7) \\
\text { Depression }(>7)\end{array}$ & $\begin{array}{l}81(80.2) \\
20(19.8)\end{array}$ & $\begin{array}{l}90.0 \pm 9.5 \\
83.6 \pm 12.6\end{array}$ & & 0.036 \\
\hline \multicolumn{5}{|l|}{ ECR-M16 } \\
\hline $\begin{array}{l}\text { Anxiety } \\
\text { Avoidance }\end{array}$ & & $\begin{array}{l}3.38 \pm 1.42 \\
2.73 \pm 1.21\end{array}$ & $\begin{array}{l}-0.248 \\
-0.038\end{array}$ & $\begin{array}{l}0.016 \\
0.716\end{array}$ \\
\hline \multicolumn{5}{|l|}{ ECR-M16 groups } \\
\hline $\begin{array}{l}\text { Secure } \\
\text { Dismissing } \\
\text { Preoccupied } \\
\text { Fearful }\end{array}$ & $\begin{array}{l}22(23.2) \\
27(28.4) \\
23(24.2) \\
23(24.2)\end{array}$ & $\begin{array}{l}91.1 \pm 8.9 \\
89.6 \pm 10.1 \\
86.0 \pm 10.1 \\
87.5 \pm 12.8\end{array}$ & & 0.308 \\
\hline \multicolumn{5}{|l|}{ ECR-M16 groups } \\
\hline $\begin{array}{l}\text { Secure } \\
\text { Insecure }\end{array}$ & $\begin{array}{l}22(23.2) \\
73(76.8)\end{array}$ & $\begin{array}{l}91.1 \pm 8.9 \\
87.8 \pm 11.0\end{array}$ & & 0.231 \\
\hline MoCA score & & $16.84 \pm 7.63$ & -0.266 & 0.007 \\
\hline $\begin{array}{l}\text { Normal }(\geq 26) \\
\text { Cognitive impairment }(<26)\end{array}$ & $\begin{array}{l}12(11.9) \\
89(88.1)\end{array}$ & $\begin{array}{l}86.2 \pm 8.9 \\
89.1 \pm 10.7\end{array}$ & & 0.083 \\
\hline PHQ-15 score (somatic symptom severity) & & $7.97 \pm 5.33$ & -0.228 & 0.022 \\
\hline $\begin{array}{l}\text { Minimal } \\
\text { Low } \\
\text { Medium } \\
\text { High }\end{array}$ & $\begin{array}{l}29(28.7) \\
33(32.7) \\
27(26.7) \\
12(11.9)\end{array}$ & $\begin{array}{l}92.0 \pm 8.2 \\
90.0 \pm 7.9 \\
86.3 \pm 12.6 \\
83.0 \pm 13.5\end{array}$ & & 0.096 \\
\hline
\end{tabular}


Table 5 - Multivariate linear regression analysis for potential predictors of a higher quality of life index* among chronic hemodialysis patients.

\begin{tabular}{|c|c|c|c|c|c|c|}
\hline \multirow[t]{3}{*}{ Variables } & \multicolumn{3}{|c|}{ Unstandardized coefficients } & \multirow{3}{*}{$\begin{array}{c}\text { Standardized coefficients } \\
\text { Beta }\end{array}$} & \multirow[t]{3}{*}{ t-test } & \multirow[t]{3}{*}{$P$-value } \\
\hline & \multirow[t]{2}{*}{ Beta } & \multicolumn{2}{|c|}{$95 \%$ confidence intervals } & & & \\
\hline & & Lower & Upper & & & \\
\hline Constant & 4.720 & 4.528 & 4.912 & & 48.227 & $<0.001$ \\
\hline HAD anxiety score & -0.061 & -0.094 & -0.028 & -0.425 & -3.537 & 0.001 \\
\hline MoCA score & -0.083 & -0.146 & -0.020 & -0.301 & -2.597 & 0.013 \\
\hline Married/nonmarried & 0.080 & 0.019 & 0.141 & 0.309 & 2.563 & 0.014 \\
\hline
\end{tabular}

*Adjusted means were adjusted for age, marital status, occupation, years of hemodialysis, use of alternative medicine, problems getting to hemodialysis unit, number of comorbid diseases, cardiovascular disease, and hepatitis B and C. HAD - hospital anxiety and depression scale, MoCA - montreal cognitive assessment. R-square was 0.334; adjusted R-square was 0.288; Model F was 7.34, $p<0.001$.

QoL was generally higher (88\%) than those previously reported using the same tool with chronic hemodialysis patients in the United Arab Emirates (77\%), Jordan (67\%), and Iran (68\%). ${ }^{32-34}$ Interestingly, the results of these studies followed the same pattern observed in the current study, where family and psychological/ spiritual domains received the highest domain scores. Researchers have reported that the conservative culture in KSA, characterized by strong family bonds, social support, and religious beliefs, positively impacts QoL in chronic dialysis patients. ${ }^{16,35}$

The burden of ESRD disease and its management reportedly increase the risk of stress and psychosocial disorders. ${ }^{36}$ The chronic hemodialysis patients in the current study generally had a better than expected psychosocial profile, which may have contributed to the high QoL level observed in the current study. Approximately $20-25 \%$ of these patients had depressive symptoms, and $16 \%$ had anxiety symptoms. These figures are considerably lower than those previously reported for chronic hemodialysis patients in KSA. ${ }^{18,37,38}$ For example, using HAD scores of $\geq 8$, $45-52 \%$ of chronic hemodialysis patients in Makkah and Al-Madinah, KSA, had depressive symptoms, and $40-42 \%$ had anxiety symptoms. ${ }^{18,37}$ Actually, the current figures for depressive and anxiety symptoms are similar to the levels described in primary care patients in KSA. ${ }^{39,40}$ The majority of current patients were very satisfied with both the hemodialysis care provided and the social and logistic support received. Additionally, religious and cultural beliefs among chronic hemodialysis patients in KSA positively impact overall psychological functioning. ${ }^{35}$ These beliefs can change the way the patient perceives the suffering and hardship associated with the disease and the commitment to its management. ${ }^{41}$

Although most of the current patients had impaired cognitive function, it is still better than previously reported. ${ }^{20,42}$ For example, the chronic hemodialysis patients examined in Jeddah, KSA, had $>35$ out of 36 points on the blessed dementia information memory concentration test, with almost all patients having cognitive impairment. ${ }^{42}$ Cognitive impairment has been linked to aging, depression, and stroke. ${ }^{43,44}$ Current patients were relatively young, had a very low stroke burden $(2 \%)$ and had a lower than expected level of depressive symptoms. Almost three-fourths of the current patients had an insecure attachment style. This was probably a result of higher anxiety symptoms $(3.4$ points) rather than avoidance (2.7 points). It is difficult to compare this finding, as we could not identify local or international studies estimating attachment style in chronic hemodialysis patients. Finally, approximately $40 \%$ of the current patients reported medium to high severity of somatic symptoms. While this result is probably expected in chronic hemodialysis patients with multiple comorbidities, we could not identify local studies estimating the severity of somatic symptoms in chronic hemodialysis patients in KSA. Consistent with this finding, the lowest QoL score for the current patients was in the health and functioning domain. Nevertheless, the severity of somatic symptoms in the current study was very similar to that for Saudi patients with diabetes. ${ }^{45}$

With exception of cognitive impairment, the scores of all psychosocial disorders examined in the current study were negatively correlated with QoL in univariate analysis. These included depressive and anxiety 
symptoms, insecure attachment style, and severe somatic symptoms. Other studies report similar findings for chronic dialysis patients internationally, especially for depression, anxiety, and stress. ${ }^{12-14}$ We could not identify any local studies focusing on the impact of psychosocial disorders on QoL among chronic hemodialysis patients in KSA. However, psychosocial disorders, especially depressive and anxiety symptoms, were associated with poor QoL among patients with diabetes, skin disease, and sickle cell disease. ${ }^{45-47}$

Anxiety symptoms and cognition level (better cognition) in the current study remained negatively associated with QoL in multivariate analysis. Consistently, in a review of 5 studies that examined the role of both anxiety and depression on QoL, anxiety alone was an independent predictor of $\mathrm{QoL}$ in 2 studies, both anxiety and depression were independent predictors in 2 studies, and depression alone was an independent predictor in one study. ${ }^{12}$ The disappearance of depressive symptoms in the multivariate analysis in the current study may be related to its strong correlation with anxiety symptoms $(\mathrm{r}=0.577, p<0.001)$. Several studies pointed to the negative impact of anxiety on psychosocial functioning in several functional domains. ${ }^{48}$ Additionally, anxiety is prone to affect the mental health dimensions of health-related QoL. ${ }^{12}$ Unlike the majority of the studies, patients who were not cognitively impaired were more negatively affected in QOL. ${ }^{13,48}$ Consistent with this finding, some studies found that normal cognition may increase the negative impact of comorbidity on QOL. ${ }^{49}$ It is not clear why the presence of cognitive impairment was associated with less negative impact on QOL, despite, it would be expected that given better cognition is associated with better self-care, however there is a possibility that cognitive impairment may reduce insight.

With the exception of marital status, the sociodemographic and clinical characteristics of the current patients did not play a major role in QoL. The positive role of marital status could relate to the caregiver role of the partner. Additionally, married chronic hemodialysis patients tend to perform better in physical QoL scores. ${ }^{50}$ However, local studies among chronic hemodialysis patients could not detect any significant impact of marital status. ${ }^{15,16}$ Studies that examined the role of psychosocial factors in QoL using multivariate analysis could not detect major roles for sociodemographic and clinical characteristics. $32,51,52$

The current study examined the associations of psychosocial predictors with QoL in chronic hemodialysis patients. The study methodology involved the examination of patients from 3 centers using multiple validated psychosocial tools, and the use of multivariate analysis.

Study limitations. A number of limitations can be recognized. The cross-sectional design cannot confirm causal association between psychosocial predictors and QoL. The convenience sampling may hinder the generalization of the current findings to all chronic hemodialysis patients in KSA. The lack of local studies compromised the ability to compare some findings such as attachment style and severity of somatic symptoms. However, the findings provide an opportunity for researchers to confirm or reject them in the future. The use of multiple psychological tools may negatively impact the enthusiasm and accuracy of the patient response. However, as they have been adjusted for in the multivariate analysis, the impact on the findings should be minimal.

In conclusion, we report a high overall QoL and relatively better psychosocial profiles among patients receiving hemodialysis in Riyadh, KSA. Psychosocial disorders were negatively correlated with QoL in univariate analysis. Also, patients who were not cognitively impaired were more negatively affected in QOL. Anxiety symptoms and better cognition level remained negatively associated with QoL in multivariate analysis. The findings of the current study re-emphasize the importance of the early detection and management of psychosocial disorders in chronic hemodialysis patients to improve QoL.

Acknowledgment. The authors gratefully acknowledge the College of Medicine Research Center, Deanship of Scientific Research, King Saud University, Riyadh, Kingdom of Saudi Arabia for supporting this study. Furthermore, the authors express their gratitude to Dr. Aiman El-Saed for his assistance in data analysis.

\section{References}

1. Luyckx VA, Tonelli M, Stanifer JW. The global burden of kidney disease and the sustainable development goals. Bull World Health Organ 2018; 96: 414D-422D.

2. Liyanage T, Ninomiya T, Jha V. Worldwide access to treatment for end-stage kidney disease: A systematic review. J Vasc Surg 2015; 62: 1089.

3. White SL, Chadban SJ, Jan S, Chapman JR, Cass A. How can we achieve global equity in provision of renal replacement therapy? Bull World Health Organ 2008; 86: 229-237.

4. Mushi L, Marschall P, Fleßa S. The cost of dialysis in low and middle-income countries: a systematic review. BMC Health Serv Res 2015; 15: 506.

5. Al-Sayyari AA, Shaheen FA. End stage chronic kidney disease in Saudi Arabia. A rapidly changing scene. Saudi Med J 2011; 32: 339-346.

6. Saudi Center for Organ Transplantation (SCOT). Dialysis in the Kingdom of Saudi Arabia. Saudi J Kidney Dis Transplant 2018; 29: 1012-1020. 
7. Feroze U, Martin D, Kalantar-Zadeh K, Kim JC, Reina-Patton A, Kopple JD. Anxiety and depression in maintenance dialysis patients: preliminary data of a cross-sectional study and brief literature review. J Ren Nutr 2012; 22: 207-210.

8. Semaan V, Noureddine S, Farhood L. Prevalence of depression and anxiety in end-stage renal disease: A survey of patients undergoing hemodialysis. Appl Nurs Res 2018; 43: 80-85.

9. van Zwieten A, Wong G, Ruospo M, Palmer SC, Barulli M, Iurillo A, et al. Prevalence and patterns of cognitive impairment in adult hemodialysis patients: the COGNITIVE-HD study. Nephrol Dial Transplant 2018; 33: 1197-1206.

10. Schouten RW, Haverkamp GL, Loosman WL, Shaw PKC, van Ittersum FJ, Smets YFC, et al. Anxiety symptoms, mortality, and hospitalization in patients receiving maintenance dialysis: A cohort study. Am J Kidney Dis 2019; 74: 158-166.

11. Drayer RA, Piraino B, Reynolds CF 3rd, Houck PR, Mazumdar S, Bernardini J, et al. Characteristics of depression in hemodialysis patients: symptoms, quality of life and mortality risk. Gen Hosp Psychiatry 2006; 28: 306-312.

12. García-Llana H, Remor E, Del Peso G, Selgas R. The role of depression, anxiety, stress and adherence to treatment in dialysis patients health-related quality of life: a systematic review of the literature. Nefrologia 2014; 34: 637-657.

13. Chan R, Brooks R, Steel Z, Heung T, Erlich J, Chow J, et al. The psychosocial correlates of quality of life in the dialysis population: a systematic review and meta-regression analysis. Qual Life Res 2012; 21: 563-580.

14. Cangini G, Rusolo D, Cappuccilli M, Donati G, La Manna G. Evolution of the concept of quality of life in the population in end stage renal disease. A systematic review of the literature. Clin Ter 2019; 170: e301-e320.

15. AL-Jumaih A, Al-Onazi K, Binsalih S, Hejaili F, Al-Sayyari A. A study of quality of life and its determinants among hemodialysis patients using the KDQOL-SF instrument in one center in Saudi Arabia. Arab J Nephrol Transplant 2011; 4: 125-130.

16. Al Wakeel J, Al Harbi A, Bayoumi M, Al-Suwaida K, Al Ghonaim M, Mishkiry A. Quality of life in hemodialysis and peritoneal dialysis patients in Saudi Arabia. Ann Saudi Med 2012; 32: 570-574.

17. AlShahrani MA, Alayed ASM, AlShehri AH, Solaiman O, Awadalla NJ, Alhomrany M. Depression and impaired work productivity among hemodialysis patients in south region of Saudi Arabia. Saudi J Kidney Dis Transpl 2018; 29: 1133-1138.

18. Mosleh H, Alenezi M, Al Johani S, Alsani A, Fairaq G, Bedaiwi R. Prevalence and factors of anxiety and depression in chronic kidney disease patients undergoing hemodialysis: A crosssectional single-center study in Saudi Arabia. Cureus 2020; 12 : e6668.

19. Cruz JP, Colet PC, Alquwez N, Inocian EP, Al-Otaibi RS, Islam SM. Influence of religiosity and spiritual coping on healthrelated quality of life in Saudi haemodialysis patients. Hemodial Int 2017; 21: 125-132.

20. Al Zaben F, Khalifa DA, Sehlo MG, Al Shohaib S, Shaheen F, Alhozali $\mathrm{H}$, et al. Depression in patients with chronic kidney disease on dialysis in Saudi Arabia. Int Urol Nephrol 2014; 46: 2393-2402.

21. Al-Homrany MA, Bilal AM. Psycho-social features of chronic dialysis patients in Saudi Arabia: Experience of one centre. Saudi J Kidney Dis Transpl 2001; 12: 164-171.
22. AlHadi AN, AlAteeq DA, Al-Sharif E, Bawazeer HM, Alanazi $\mathrm{H}$, AlShomrani AT, et al. An arabic translation, reliability, and validation of patient health questionnaire in a Saudi sample. Ann Gen Psychiatry 2017; 16: 32.

23. Becker S, Al Zaid K, Al Faris E. Screening for somatization and depression in Saudi Arabia: a validation study of the PHQ in primary care. Int J Psychiatry Med 2002; 32: 271-283.

24. Malasi TH, Mirza IA, el-Islam MF. Validation of the hospital anxiety and depression scale in Arab patients. Acta Psychiatr Scand 1991; 84: 323-326.

25. Lo C, Walsh A, Mikulincer M, Gagliese L, Zimmermann C, Rodin G. Measuring attachment security in patients with advanced cancer: psychometric properties of a modified and brief experiences in close relationships scale. Psychooncology 2009; 18: 490-499.

26. Alosaimi FD, Asiri M, Alsuwayt S, Alotaibi T, Bin Mugren M, Almufarrih A, et al. Psychosocial predictors of nonadherence to medical management among patients on maintenance dialysis. Int J Nephrol Renovasc Dis 2016; 9: 263-272.

27. Fraley RC, Waller NG, Brennan KA. An item response theory analysis of self-report measures of adult attachment. J Pers Soc Psychol 2000; 78: 350-365.

28. Rahman TT, El Gaafary MM. Montreal cognitive assessment Arabic version: reliability and validity prevalence of mild cognitive impairment among elderly attending geriatric clubs in Cairo. Geriatr Gerontol Int 2009; 9: 54-61.

29. Kroenke K, Spitzer RL, Williams JB. The PHQ-15: validity of a new measure for evaluating the severity of somatic symptoms. Psychosom Med 2002; 64: 258-266.

30. Ferrans CE, Powers MJ. Quality of life index: development and psychometric properties. ANS Adv Nurs Sci 1985; 8: 15-24.

31. Halabi JO. Psychometric properties of the Arabic version of quality of life index. J Adv Nurs 2006; 55: 604-610.

32. Ayoub AM, Hijjazi KH. Quality of life in dialysis patients from the United Arab Emirates. J Family Community Med 2013; 20: 106-112.

33. Nabolsi MM, Wardam L, Al-Halabi JO. Quality of life, depression, adherence to treatment and illness perception of patients on haemodialysis. Int J Nurs Pract 2015; 21: 1-10.

34. Rambod M, Rafii F. Perceived social support and quality of life in Iranian hemodialysis patients. J Nurs Scholarsh 2010; 42: 242-249.

35. Al Zaben F, Khalifa DA, Sehlo MG, Al Shohaib S, Binzaqr SA, Badreg AM, et al. Religious involvement and health in dialysis patients in Saudi Arabia. J Relig Health 2015; 54: 713-730.

36. Feroze U, Martin D, Reina-Patton A, Kalantar-Zadeh K, Kopple JD. Mental health, depression, and anxiety in patients on maintenance dialysis. Iran J Kidney Dis 2010; 4: 173-180.

37. Turkistani I, Nuqali A, Badawi M, Taibah O, Alserihy O, Morad M, et al. The prevalence of anxiety and depression among end-stage renal disease patients on hemodialysis in Saudi Arabia. Ren Fail 2014; 36: 1510-1515.

38. AlDukhayel A. Prevalence of depressive symptoms among hemodialysis and peritoneal dialysis patients. Int J Health Sci (Qassim) 2015; 9: 9-16.

39. Al-Qadhi W, Ur Rahman S, Ferwana MS, Abdulmajeed IA. Adult depression screening in Saudi primary care: prevalence, instrument and cost. BMC Psychiatry 2014; 14: 190.

40. AlKhathami AD, Alamin MA, Alqahtani AM, Alsaeed WY, AlKhathami MA, Al-Dhafeeri AH. Depression and anxiety among hypertensive and diabetic primary health care patients. Could patients' perception of their diseases control be used as a screening tool? Saudi Med J 2017; 38: 621-628. 
41. Patel SS, Shah VS, Peterson RA, Kimmel PL. Psychosocial variables, quality of life, and religious beliefs in ESRD patients treated with hemodialysis. Am J Kidney Dis 2002; 40: 1013-1022.

42. Ibrahim S, Hossam M, Belal D. Study of non-compliance among chronic hemodialysis patients and its impact on patients' outcomes. Saudi J Kidney Dis Transpl 2015; 26: 243-249.

43. Jung S, Lee YK, Choi SR, Hwang SH, Noh JW. Relationship between cognitive impairment and depression in dialysis patients. Yonsei Med J 2013; 54: 1447-1453.

44. Pereira AA, Weiner DE, Scott T, Sarnak MJ. Cognitive function in dialysis patients. Am J Kidney Dis 2005; 45: 448-462.

45. Alosaimi FD, Labani R, Almasoud N, Alhelali N, Althawadi L, AlJahani DM. Associations of foot ulceration with quality of life and psychosocial determinants among patients with diabetes; a case-control study. J Foot Ankle Res 2019; 12: 57.

46. Adam SS, Flahiff CM, Kamble S, Telen MJ, Reed SD, De Castro LM. Depression, quality of life, and medical resource utilization in sickle cell disease. Blood Adv 2017; 1: 1983-1992.
47. Ahmed AE, Al-Dahmash AM, Al-Boqami QT, Al-Tebainawi YF. Depression, anxiety and stress among Saudi Arabian dermatology patients: cross-sectional study. Sultan Qaboos Univ Med J 2016; 16: e217-e223.

48. Mendlowicz MV, Stein MB. Quality of life in individuals with anxiety disorders. Am J Psychiatry 2000; 157: 669-682.

49. Gallagher R, Sullivan A, Burke R, Hales S, Sharpe P, Tofler G. Quality of life, social support and cognitive impairment in heart failure patients without diagnosed dementia. Int J Nurs Pract 2016; 22: 179-188.

50. Ikonomou M, Skapinakis P, Balafa O, Eleftheroudi M, Damigos $\mathrm{D}$, Siamopoulos KC. The impact of socioeconomic factors on quality of life of patients with chronic kidney disease in Greece. J Ren Care 2015; 41: 239-246.

51. Kring DL, Crane PB. Factors affecting quality of life in persons on hemodialysis. Nephrol Nurs J 2009; 36: 15-55.

52. Cukor D, Coplan J, Brown C, Friedman S, Newville H, Safier $\mathrm{M}$, et al. Anxiety disorders in adults treated by hemodialysis: a single-center study. Am J Kidney Dis 2008; 52: 128-136. 